\title{
Enhancing Services Selection by Using Non-Functional Properties within BPMN in SOA Context
}

\author{
Sarah Zribi ${ }^{1,2}$, Frédérick Bénaben ${ }^{1}$, Jean-Pierre Lorré ${ }^{2}$, and Hervé Pingaud ${ }^{3}$ \\ ${ }^{1}$ Ecole des Mines d'Albi-Carmaux, Campus Jarlard, \\ Route de Teillet, 81000 Albi, France \\ \{szribi, frederick.benaben\}@mines-albi.f \\ ${ }^{2}$ Linagora, 75 Route de Revel, 31400 Toulouse, France \\ \{szribi, jplorre\} @linagora.com \\ ${ }^{3}$ Université Jean-François Champoilion, Place de Verdun, 8100 Albi, France \\ pingaud@univ-jfc.fr
}

\begin{abstract}
In the Future Internet vision, multiple services coming from heterogeneous organizations have to collaborate together in order to achieve the customers' demands from both functional and non-functional point of view. Hence, it is necessary, within an organization, to put in place an interoperable approach that ensures the best functioning control and selection of services. This paper presents a comprehensive framework for representing the customer Non-Functional Properties (NFP) within a collaborative Business Process Management (BPM) and the contribution of the Service Oriented Architecture Governance (SOA Governance) to give customers a better selection of services that best suits their business NFPs requirements among all Web Services candidates provided by functional matching.
\end{abstract}

Keywords: Collaborative Process, Interoperability, Non Functional Properties, Service Selection, SOA Governance, BPMN, SLA.

\section{Introduction}

In an era marked by a deep and growing technological progress, the environment in which firms are located has become more complex and characterized by increasing competition. Companies have realized that they can ensure their future development using new technologies such as Service-Oriented Architecture (SOA) and by migrating their Information System to this paradigm. SOA is a computer system architectural style based on distributed and loosely coupled applications in which functions are separated into distinct web services. A Web Service (WS) can be defined as an autonomous and distributed entity, independent of any platform and that responds to a particular need. However, to achieve the user's requirements, several heterogeneous WS can be assembled to interact and collaborate within a business process[1]. Selecting the right WS in each task of this business process has become a major challenge in a collaborative context. On one hand, this is due, to the inexorable rise of services existing in the web. On the other hand, it is also due to the fact that the number of services offering the similar functional properties is relatively huge. That is why the NFPs play a crucial role to sort them. 
Our Contribution. To address the aforementioned challenge, this paper takes into account these properties with a particular focus on Non-Functional ones. It presents a complete framework for modeling NFPs annotations within a Business Process Management Notation (BPMN) 2.0, discovering WS, selecting and ranking them. The contribution of this paper is threefold. First, we propose a solid way to annotate NFPs in BPMN. Since interoperability is an important factor in SOA, it is essential to ease it by adopting standards. Thus, we are principally interested in the use of common agreed standards for the NFPs annotations in BPMN. We also incorporate a graphical representation of these NFPs annotations to enable the user to annotate his NFPs requirements in each task of the BPMN2.0 process. Second, an essential component in our approach is the SOA Governance Registry. It provides an uniform way of governing WS. We use the SOA Governance Framework to facilitate and standardize the discovery of WS and their NFPs. Our third contribution binds the two previously ones. We propose algorithms that match BPMN business tasks with the set of WS candidates published in SOA Governance registry and provided by semantic matching according to the NFPs requirements of the user.

Related Work. BPMN, non-functional properties and WS selection are very active research topics. Guangjun and al., in [1], present a method for WS selection based on ontology to select the best WS from a functional and QoS (Quality of Services) points of view. However, their NFPs selection approach never takes into account the user's NFPs preferences values. Their QoS filter consists on ranking services candidates between them according only to the user's NFPs weights. Youakim and al. propose, in [2] a framework for selecting WS based on the user's NFPs. Even if they take into account the user's preferences, they do not use a common well-known model for representing NFPs. Besides, they don't detail the registry where WS are published. In [3], John and al. also present a framework that details how to represent and use NFPs within a process. In contrast, their work enumerates only some NFPs and they mostly focus on representing them in the development phase and not in the entire life-cycle. The key difference between previous approaches and our solution is that we take into account all the user's preferences in the selection and ranking of the set of WS provided by the semantic functional matching. Thus, the result is very close and fits the user's expectations. What's more, none of those previous approaches does address the issue of a robust registry where the discovery of WS is done. The rest of the paper is structured as follows. First, in Section 2 we present an overview of the important techniques related to our approach. Then, the architecture of our approach is detailed in the Section 3. Finally, a conclusion and future works are presented in Section 4.

\section{Preliminaries}

This section briefly outlines the major concepts and techniques used in our approach.

BPMN is a standard supported by the Object Management Group (OMG). It allows the design of a wide variety of business processes, the design of diagrams, which are easily understandable and potentially executable. The graphical modeling using BPMN is done using a quite intuitive set of elements allowing a quick understanding for any user if the diagram is not too big or too complicated.

Web Service-Policy (WS-Policy) was developed by the W3C in 2006. The basic construct around WS-Policy is the assertion, which is a bit of metadata. Assertions are 
not defined, but rather left to developers of individual standards. That's why to define NFPs assertions, we propose to combine WS-Policy with the use of the Web Service Quality Factors (WSQF) model that clearly depicts a model for NFPs.

WSQF was published in 2011 by the WS Quality Model TC as an OASIS Committee specification. According to the specification [4], WSQF refer to a set of several properties which are classified into six groups: 1-Business Value Quality (refers to the business perspective to evaluate the business value of WS), 2-Service Level Measurement (characterize the quality that a user perceives when actually using WS), 3-Interoperability (represents the ability to evaluate whether a WS system conforms to standards), 4-Business Processing (indicates the capability of a WS platform for assuring correctness in business processing), 5-Manageability (delineates the capability of a WS platform for assuring correctness in business processing), and 6-Security (identifies the degree of ability that can protect WS from various threats).

Based on this, WSQF model comprises the majority of NFPs that can concern a WS during its entire life-cycle. Which resonates perfectly with our needs.

SOA Governance, according to definitions presented in [6], can be defined as a set of processes, rules, mechanisms of control, enforcement policies and best practices put in place to ensure the successful achievement of the SOA. Its central component is the registry that promotes the discovery and reusability of WS. As detailed in [6] and [6], SOA Governance registries serve not only to catalog and inventory data, but also as places to store metadata about services as the descriptions of their functionalities, service contracts, capabilities and NFPs.

Service Level Agreement (SLA) is a commonly used way to indicate nonfunctional objectives to be reached by the business service once deployed. They are also needed to define the responsible entities to be alerted if a constraint is violated. SLA template is a model used for the representation of its various clauses and the main NFPs that can be measured at the runtime phase.

\section{General Architecture}

In the remainder of this section, we focus on the description of our approach. Formally, the proposed framework, as defined in Figure 1, consists of two major parts. The first one concerns the graphic representation of BPMN with NFPs Annotations. The second one defines an algorithm witch matches the user's NFPs requirements with WS that are provided from the functional matching.

Modeling NFPs Annotations in BPMN. WS is characterized by two sorts of properties: functional properties (what does the WS do?) and NFPs (how does the WS do its functionalities?). The final selection of WS depends on matching according to both of them. The functional matching, as detailed in [7], provides a set of WS that match functionally (syntactic and semantic) with the target business task request. Then, using this provided set of WS, we match them, this time, according to their NFPs which is our main interests in this section. As explained previously, we use the WSQF model that extends WS-Policy as support to such annotations. The Figure 2 shows a screenshot of our graphical framework that supports NFPs annotations at the design phase of the business process. 

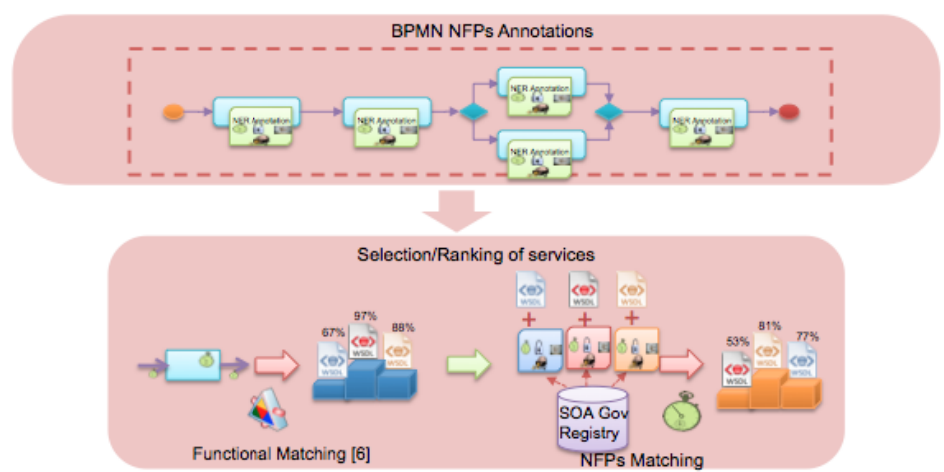

Fig. 1. General architecture of enhancing WS selection according to NFPs BPMN annotations

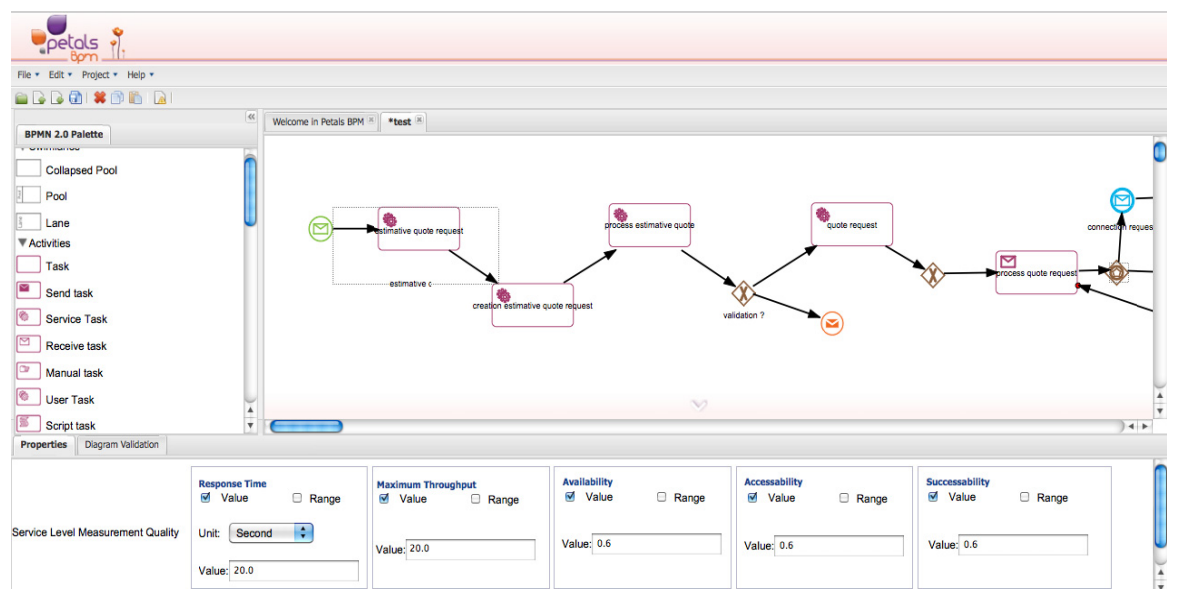

Fig. 2. Screenshot from our BPMN design framework supporting NFPs annotations

SOA Governance Registry. According to the BPMN annotated file and the algorithm of functional matching, as explained in [6], a first set of services candidates is given. For each service of this set and among to our SOA Governance Registry framework, we can extract all NFPs that correspond to it. For example the response time property can be found it in the SLA template published by the service provider. Finally, among all this information, we can apply the non-functional algorithm presented in the following.

NFP Services Selection and Ranking Algorithm. Since the non-functional features play different roles, we can identify two sorts of NFPs: those where it is better to maximize the value, such as the availability, the service recognition, the service provider reputation, etc.; and those where it is better to minimize their values like the price, the response time. We call the first set of properties "NFPs to maximize" and the second set "NFPs to minimize". Besides, NFPs are also subdivided into further levels according to the type of the property. We identify three levels of types: Numerical values, Boolean values, and Ranges. Our NFPs matching algorithm take into account this levels and is presented below. 
Step1: Elimination of Services Candidates outliers: our approach aims to be very close to the user's preference, so all services provided by the functional matching do not necessarily have to be kept in the case where they unduly deviate from the user's NFPs requirements. Hence, we set a threshold tolerance parameter (we consider the default $30 \%$ ) and we eliminate any service, from the services candidates, that at least has one NFP value that exceeds this threshold.

Step2: Normalization and Centering of Services Candidates: Since the values and dimensions of NFPs properties are different, they cannot be compared directly between them. It is necessary to normalize the NFPs values to bring all the features into proportion with one another. Once the normalization of the set of Services candidates is done, the centering part aims to represent them around the target business task. In the following, we explain clearly this steps using the adequate formula in each case.
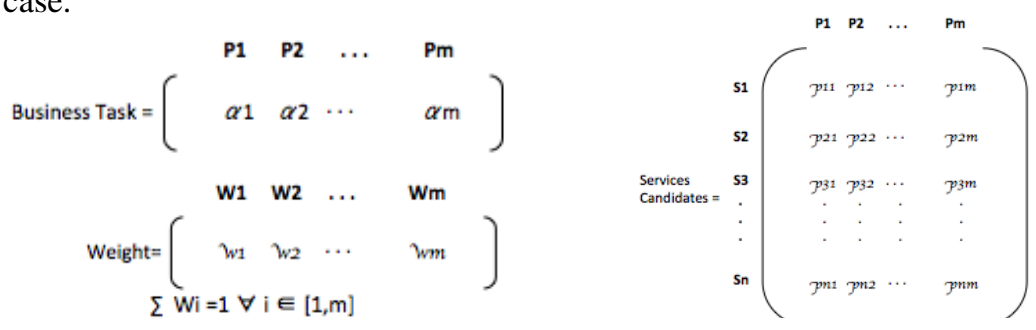

For the business task: As our main interest is to be close to the user's requirements, we normalize the values NFPs values of services candidates according to the user's preferences. Each value of the business task will be equal to 1 in the normalization part and 0 after the centering step.

$$
\text { Normalized and Centered Business Task }[\mathrm{m}]=0 \text {. }
$$

For Services Candidates: the coefficients of NFPs for each service candidate reflect meaning between the NFPs of the service and the NFP of the user requirement. We distinguish three different ways to normalize according to each type of NFPs.

A) Case of Boolean values: there is not any change because the NFPs can have only two values: 1 or 0 . Then there is not any obligation to normalize them.

B) Case of Numerical values: the normalization and centering of the value of each NFP of Service Candidates is depending on the NFPs Properties when it is a property to maximize, we use the formula (2) and the formula (2') when it is a to minimize.

Normalized and Centered Service Candidate $[\mathrm{nm}]=\frac{\text { Service Candidate }[\mathrm{nm}]}{\text { Business Task }[\mathrm{m}]}-1$.

Normalized and Centered Service Candidate $[\mathrm{nm}]=1-\frac{\text { Service Candidate }[\mathrm{nm}]}{\text { Business Task }[\mathrm{m}]}$.

However, there is a particular case when the value of NFP in Business Task is equal to zero. So in this case, for each non-zero NFP of services candidates, we apply 
the formula (3) if it NFP to maximize and (3') in the other way.

$$
\begin{aligned}
& \text { Normalized and Centered Service Candidate }[\mathrm{nm}]=\frac{\text { Service Candidate }[\mathrm{nm}]}{\operatorname{Max}(\text { Service Candidate }[\mathrm{m}])} . \\
& \text { Normalized and Centered Service Candidate }[\mathrm{nm}]=-\frac{\text { Service Candidate }[\mathrm{nm}]}{\operatorname{Max}(\text { Service Candidate }[\mathrm{m}])} .
\end{aligned}
$$

C) Case of Ranges in the Business Task: for each NFP value in the services candidates that is includes in the range of corresponding NFP in business task, satisfy the requirement of the user on this NFP. The value of NFP for each service candidate after the normalization and centering is calculated according to (4) if it is NFP to maximize and (4') else.

$$
\begin{aligned}
& \text { Normalized and } \\
& \text { Centered Service } \\
& \text { Candidate }[\mathrm{nm}]= \\
& \text { Service Candidate[nm]-Business Task [m] Lower bound } \\
& \text { Business Task[m]Upper Bound-Business Task[m]Lower Bound . } \\
& \frac{\text { Business Task }[\mathrm{m}] \text { Upper bound-Service Candidate }[\mathrm{nm}]}{\text { Business Task[m] Upper Bound-Business Task[m]Lower Bound }} \text {. }
\end{aligned}
$$

Else, when the NFP value is out of the range, it is normalize with the upper bound when it is superior -formula (5)- and, else, with the lower one- formula (5').

Normalized Service Candidate $[\mathrm{nm}]=\frac{\text { Service Candidate }[\mathrm{nm}]}{\text { Business Task[m]Upper Bound }}$.

Normalized Service Candidate $[\mathrm{nm}]=-\frac{\text { Service Candidate }[\mathrm{nm}]}{\text { Business Task }[\mathrm{m}] \text { Lower Bound }}$.

As previously the centering of NFPs values depends on where it is a property to maximize, then we use the formula (6) or to minimize, then we use the formula (6').

Normalized and Centered Service Candidate [nm] = Normalized Service Candidate [nm]-1 .

Normalized and Centered Service Candidate $[\mathrm{nm}]=1$ - Normalized Service Candidate [nm] .

\section{Step3: Selection and Ranking Services Candidates basing on to the NFPs values}

At this step, we have a matrix of services candidates with normalized and centered NFPs values. We distinguish three types of NFPs values: positive ones (that means that the NFP value acts positively towards the corresponding user's NFP requirement), null ones (that means that the NFP value is exactly the same as the user one), and finally negative ones (in this case, NFP value acts negatively towards the corresponding user's NFP requirement). Indeed, each NFP value of this matrix reflects the degree to which it influences the user's requirements.

To tackle with that, we propose to divide the matrix into two sub-matrixes. The first one includes services candidates that have all NFPs values equal to or greater than zero. The second sub-matrix contains the rest of services candidates. For those 
services in the first sub-matrix 1, we are sure that all of them match the user's NFPs requirements. Thus, for sorting them we need to calculate the weighted distance (according to the weights of NFPs defined by the user and that express his preferences) of each service candidate and then to rank them starting with the one who rolls the highest value. Once we classify the entire services candidates of the first sub-matrix, we address the second one. In the first step, for each positive NFP value, we replace it by zero, we calculate the weighted distance for each WS, and the rank them starting with the lowest value. In case of ex-aequo, we addition to the founded distance the weighted distance of NFPs positives values and finally we rank them starting with the higher global distance.

Illustrative Example: In the following example, we try to match a Business Task (BT) from our BPMN process once the user annotated it with his NFPs constraints. As shown in the vector «Business Task», we consider that the user annotated this BT with four NFPs: response time (in milliseconds), availability (a float value expressed by a percentage), price (in milliseconds) and encryption (is a boolean value). Response Time and Price are properties to minimize. Availability and Encryption are properties to maximize. WS Candidates corresponds to the six services provided by the functional matching. The NFPs values of these WS are expressed in the matrice « Services Candidates ».

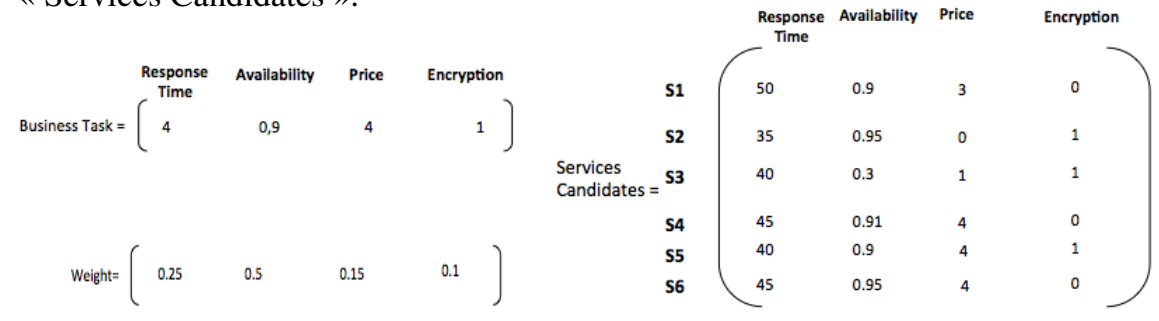

According to the step 1 of the algorithm, we deduce that the service candidate "S3" is an outlier because its availability value is out the threshold tolerance. Thus, it is necessary to eliminate it. The second step of the algorithm is normalization and centering. Thus, for the target business task, we apply the formula (1) and we obtain the vector "Normalized and Centered Business Task" below. Then, for Services Candidates, we apply the formula (2') for Response time and Price, the formula (2) for Availability and finally for Encryption we keep the same values as it is a Boolean NFP as shown below.

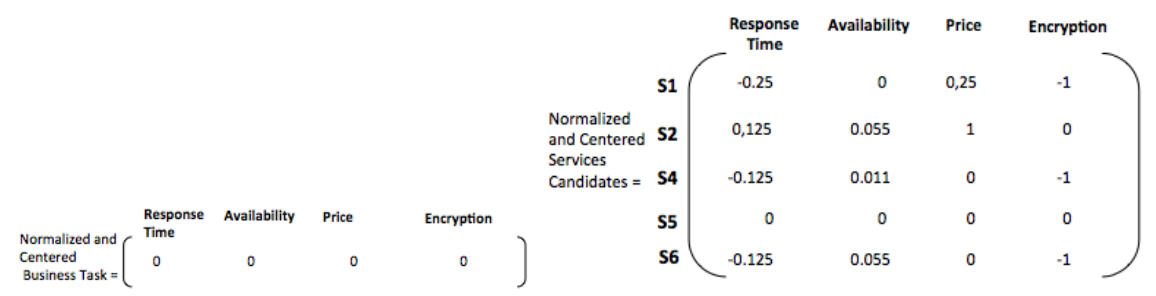


Once the business task and the services candidates are normalized and centered, the third step of our algorithm is to select and rank these services according to their degree of NFPs matching with the target business task. As we detailed previously, we divide services candidates into two sub-matrixes: the first one includes services candidates that have all NFPs values equal to or greater than zero, so in our example S2 and S5 and S1, S4 and S6 belong to the second one. Then, for the first sub-matrix (S2 and S5), to rank services we need to take into account the weights defined by the user to calculate the weighted distance as depicted in the sub-matrix below:

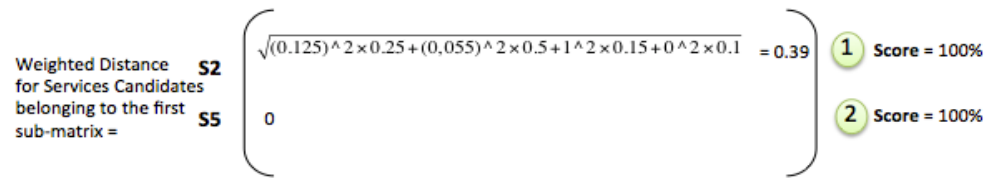

Likewise, for those that belong to the second sub-matrix (S1, S4 and S6), we obtain the following matrix:

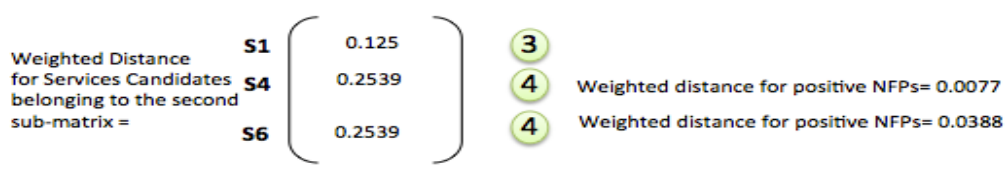

Finally, the final selecting and ranking of services candidates that best match with NFPs user requirements defined in the business task is: S2, S5, S1, S6, S4.

\section{Conclusion}

In this paper, we present a complete solution for enhancing WS selection with a particular focus on NFPs according to the NFPs BPMN business tasks requirements. Indeed, we try to match each business task annotated NFPs by the user with NFPs of the set of WS provided by the semantic matching. Thus, we consider that for one business task, there is a set of services that each meets fully the functional requirement of the user (1-1 approach). However, in many cases services need to collaborate together to achieve the requirements of the Business Task. Thus, for one business task, a collaboration of several services is required (1-n approach). We have started working on the extension of our solution by adding the 1-n matching.

Acknowledgement. This paper is based on an ongoing work supported by the Agence Nationale pour la Recherche (ANR) through the Project SocEDA: SOCial Event Driven Architecture.

\section{References}

1. Earl, T.: Service-oriented Architecture: Concepts, Technology, and Design. Prentice Hall, Englewood Cliffs (2005)

2. Guangjun, G.: A Methos for Semantic Web Service Selection Based on QoS Ontology. Journal of Computers 6(2) (2011) 
3. Badr, Y., Abraham, A., Biennier, F., Grosan, C.: Enhancing Web Service Selection by User Preferences of Non-Functional Features. In: NWESP 2008 (2008)

4. Mylopoulos, J., Chung, L., Nixon, B.: Representing and Using Non-Functional Requirements: A Process-Oriented Approach. Transactions on Software Engineering (1992)

5. Web Service Quality Factors (WSQF) Specification, http://docs.oasisopen.org/wsqm/wsqf/v1.0/WS-Quality-Factors-v1 .0-cd02 .html

6. Zribi, S., Benaben, F., Ben-Hamida, A., Lorré, J.-P.: Towards a Service and Choreography Governance Framework. In: I-ESA (2012)

7. Lesbegueries, J., Ben-Hamida, A., Salatgé, N., Zribi, S., Lorré, J.-P.: Multilevel EventBased Monitoring Framework for the Petals Enterprise Bus. In: Debs 2012, pp. 48-57 (2012)

8. Benaben, F., Boissel-Dallier, N., Pingaud, H., Lorre, J.-P.: Semantic issues in model-driver management of information system interoperability. IJCIM (2012) 